health record databases were linked and aggregated, resulting in a retrospective cohort of 727,091 adult men from 1990 to 2013. Self-reported MSM status ('gold-standard') from the three disease case surveillance databases was used to develop a multivariable prediction model for identifying MSM in the larger cohort. Models were selected using 'elastic-net' (combination of lasso and ridge regression), implemented through the GLMNet package in $\mathrm{R}$, and a final model optimized area under the receiver operating characteristics curve (AUC).

Results History of gonorrhea and syphilis diagnoses, HIV tests in the past year, history of visit to identified gay and bisexual men's clinics, and residence in MSM-dense neighborhoods (based on self-reported MSM) were all positively associated with being MSM. The selected model had a sensitivity of $72 \%$, specificity of $94 \%$, and AUC of $92 \%$. Combining selfreported MSM $(n=6,280)$ and predicted MSM $(n=85,521)$, a total of 91,801 men (13\% of the cohort) were classified as MSM.

Conclusion Applying a computational phenotyping method to administrative data yielded a cohort of 85,521 MSM, which may be used to monitor and evaluate health outcomes and healthcare utilization. Sensitivity and specificity of this model were comparable to interviewer-administered self-report measures of sexual orientation.

Disclosure No significant relationships.

\section{P564 OUTCOMES OF ANAL HPV SCREENING IN HIV+ MEN WHO HAVE SEX WITH MEN ATTENDING HIGH RESOLUTION ANOSCOPY CLINIC IN CANADA}

${ }^{1}$ Angel Chu*, ${ }^{2}$ Petra Smyczek, ${ }^{3}$ Michael Gill. ${ }^{1}$ Alberta Health Services, Calgary STI Clinic, Calgary, Canada; ${ }^{2}$ Alberta Health Services, STI Services, Edmonton, Canada; ${ }^{3}$ Alberta Health Services, Southern Alberta Clinic, Calgary, Canada

\subsection{6/sextrans-2019-sti.638}

Background Rates of anal cancer from HPV infections are significantly higher in HIV-positive men-who-have-sex-withmen (MSM) compared to the general population. Recent estimates in the US and Europe report the incidence of anal cancer amongst HIV-positive individuals to be $75-137$ cases/ 100000 person-years, a rate 30-100 times higher than the general population. Despite the restoration of immune function with modern antiretroviral therapy, the incidence of anal cancers is increasing amongst HIV-positive MSM. We report the results of an anal cancer screening program in HIV-positive MSM in Calgary, AB, Canada from October 2014 to December 2018.

Methods HIV-positive MSM attending the Southern Alberta Clinic and STI Clinic were offered an anal pap smear. Patients with abnormal cytology were examined with digital anorectal exam and High Resolution Anoscopy (HRA) to detect anal intraepithelial neoplasia (AIN). Dysplastic areas were biopsied and sent for histopathology. High-grade AIN II-III were treated using infrared coagulation.

Results A total of 214 patients were examined with HRA. 87 patients (40.7\%) had biopsy-proven AIN I, II, or III. Asymptomatic internal anal warts (AIN I) were detected in 60 patients (28\%). Twenty-five patients had AIN II-III (11.7\%) and 2 patients had invasive squamous cell cancer. High-risk oncogenic HPV was identified in $100 \%$ of the biopsy specimens.

Conclusion High rates of anal HPV infections causing AIN I, II, and III were identified in HIV-positive MSM, supporting ongoing use of HRA for anal cancer screening programs in this population. High rates of asymptomatic internal anal warts were incidentally found. These rates of anal HPV infections support consideration of offering HPV vaccination to HIV-positive MSM on clinic intake as a preventative tool.

Disclosure No significant relationships.

\section{P565 PERSONALIZED COGNITIVE COUNSELING (PCC) TO REDUCE HIV RISK FOLLOWING RECTAL GONORRHEA/ CHLAMYDIA DIAGNOSIS AMONG MSM IN PERU}

${ }^{1}$ Jesse Clark, ${ }^{2}$ Susan Chavez-Gomez, ${ }^{2}$ Angelica Castaneda-Huerta, ${ }^{3}$ Ryan Passaro, ${ }^{2}$ Williams Gonzales-Saavedra, ${ }^{2}$ Eduardo Cachay, ${ }^{2} \mathrm{~A}$ Barrantes, ${ }^{4}$ Francisco Nanclares, ${ }^{4}$ James Dilley, ${ }^{2}$ Robinson Cabello. ${ }^{1}$ UCLA Geffen School of Medicine, Medicine/Infectious Diseases, Los Angeles, USA; ${ }^{2}$ Asociacion Civil Via Libre, Lima, Peru; ${ }^{3}$ University of Tennessee Health Sciences Center, Memphis, USA; ${ }^{4}$ University of California-San Francisco, San Francisco, USA

\subsection{6/sextrans-2019-sti.639}

Background We piloted a bio-behavioral intervention for MSM in Peru based on rectal gonorrhea (GC)/chlamydia (CT) screening as an integrated HIV-STI prevention strategy.

Methods Between August-December 2018, we screened 605 MSM for rectal GC/CT using Aptima TMA and identified 101 cases among $469 \mathrm{HIV}$-uninfected men. Subjects were randomly assigned to traditional or Personalized Cognitive Counseling (PCC) at 3- and 6-Months. PCC session notes and Self-Justification Evaluation Instruments (SJEIs) were analyzed for thematic content. HIV/STI testing and behavioral risk assessments were repeated at 3- and 6-months. Statistical comparisons were conducted using Chi-square and Generalized Estimating Equations.

Results All participants reported reductions in condomless insertive (CIAI) and receptive anal intercourse (CRAI), with no significant differences between arms. In the Control arm, CRAI declined from a mean of 4.0 Partners $(77 \%$ of recent sexual contacts) at Baseline to $1.3(43 \%)$ at 3-Months and 0.7 $(24 \%)$ at 6-Months. CRAI in the intervention arm decreased from 2.8 Partners (58\% of contacts) to 1.1 (35\%) to 0.8 (20\%) ( $\mathrm{p} \geq 0.05)$. At 3 -Months, we identified 9 HIV infections and 24 new GC/CT cases across arms. At 6-Months, we diagnosed $2 \mathrm{HIV}$ infections and $7 \mathrm{GC} / \mathrm{CT}$ cases $(7 / 45 ; 15.6 \%)$ in the control arm $(4.4 \% ; 2 / 45)$, with $0 \mathrm{HIV}$ infections $(0 \% ; 0 /$ $46)$ and 9 GC/CT recurrences $(9 / 46 ; 19.6 \%)$ among PCC participants $(\mathrm{p} \geq 0.05)$. Thematic analysis demonstrated four common self-justifications: (1) Isolated events that don't reflect typical behavior; (2) Informal HIV status assessments based on partner appearance or behavior; (3) Fear of ruining the moment by discussing HIV; and (4) Structural barriers to condom availability during intercourse.

Conclusion Our pilot assessment supports the feasibility and acceptability of PCC for reducing HIV risk following rectal GC/CT diagnosis. Risk behavior reductions were similar between arms, though no new HIV infections were observed after PCC. Additional research is needed to apply PCC to problems of post-STI behavior change and PrEP uptake.

Disclosure No significant relationships. 